\title{
Ueber Anomalien bei der Esterbildung aus Säure und Alkohol
}

\author{
von
}

E. Proiswerk.

(22. IX. 19.)

Durch Zufall bin ich vor längerer Zeit auf eine Anomalie bei der Esterbildung aus Säure und Alkohol gestossen, für die ich zunächst keine Erklärung fand. Da sich aber allmählich eine umfassende klare Vorstellung aus dem ersten Unverständnis herausgebildet hat, möchte ich nicht mehr zögern, dieselbe der Öffentlichkeit zu übergeben.

In meinem Laboratorium war anlässlich einer grösseren Untersuchung der Diäthylmalonester ausgegangen. Da mir indes noch grössere Mengen Diäthylmalonsäure zur Verfügung standen, entschloss ich mich, die wohl früher noch von keiner Seite versuchte Veresterung dieser Säure durchzuführen. Ich zweifelte nicht an dem Erfolg, umsomehr, als ich kurz vorher eine technische Methode zur Darstellung von Malonester aus Malonsäure bezw. malonsaurem Calcium ausgearbeitet hatte, die sehr gute Ausbeuten lieferte. $\mathrm{Zu}$ meinem grossen Erstaunen aber konnte ich aus dem Reaktionsprodukt keinen Diäthylmalonester isolieren und als die Reaktionsmasse darauf bis zum Sieden erwärmt wurde, resultierte zwar eine zur Identifizierung genügende Menge eines wasserunlöslichen Öles, das sich aber als Diäthylessigester erwies.

Später hatte ich Gelegenheit, den grossen Unterschied in der Esterifizierbarkeit zwischen Malonsäure und Diäthylmalonsäure analytisch zu verfolgen. Aus den Zahlen im experimentellen Teil geht dieser Unterschied unzweideutig hervor. Malonsäure ist demnach, wie auch längst bekannt, leicht esterifizierbar, Diäthylmalonsäure dagegen gar nicht. Durch diese einzige Tatsache ist die Anschauung widerlegt, dass die Stärke der Säure der allein ausschlaggebende Faktor für deren Esterifizierbarkeit ist, denn Diäthylmalonsäure ist stärker sauer als Malonsäure ${ }^{1}$ ).

1) P. Walden, Z. ph. Ch. 8, 433 (1891) gibt an für Malonsäure: $K=0,163$ und für Diäthylmalonsäure: $\mathrm{K}=\mathbf{0 , 7 4}$. 
Der einzige prinzipielle Unterschied zwischen diesen beiden Säuren ist das Fehlen der $\alpha$-Wasserstoffatome bei der Diäthylmalonsäure. Die Methylenwasserstoffatome der Malonsäure sind aber leicht beweglich. Wenn die $\alpha$-Wasserstoffatome einer Säure für deren Esterifizierbarkeit mit verantwortlich zu machen sind, so würde das diese augenfällige Anomalie begreiflich machen. Was für eine Rolle spielen aber diese Wasserstoffatome bei der Esterbildung aus Säure und Alkohol und zu welchen Konsequenzen führt das bei der Verallgemeinerung?

Vor allen Dingen muss konstatiert werden, dass es eine geringe Zahl von Säuren gibt, die keine Wasserstoffatome in Alphastellung zum Carboxyl besitzen und doch leicht esterifizierbar sind, wie zum Beispiel die Oxalsäure und die Brenztraubensäure. Ja, diese Säuren sind sogar aussergewöhnlich leicht zu verestern $\left.{ }^{1}\right)$. Auffallend an diesen Säuren ist das starke Additionsbestreben der Carboxyl-CO-Gruppe, das sich zum Beispiel in der Neigung zur Hydratbildung kund gibt. Bekannt ist ferner, dass ein Carboxyl der Oxalsäure leicht reduzierbar ist ${ }^{2}$ ) und dass bei der Reduktion ausschliesslich nur eine Carboxylgruppe reduziert wird. Die Reduzierbarkeit der einen Carboxylgruppe ist demnach an das intakte Vorhandensein der zweiten gebunden. Während der ungesättigte Gharakter der CO-Doppelbindung einer Carboxylgruppe, d. h. deren Additionsvermögen, im allgemeinen kaum in Erscheinung tritt, ist sie bei den erwähnten Säuren offenbar durch Beeinflussung durch die Nachbargruppe viel stärker und ermöglicht verschiedene Additions-Reaktionen, die andere Säuren nicht zeigen. Es lässt sich demnach vermuten, dass die Esterbildung primär eine Additionsreaktion ist, was im Falle der Oxalsäure etwa so formuliert werden könnte:

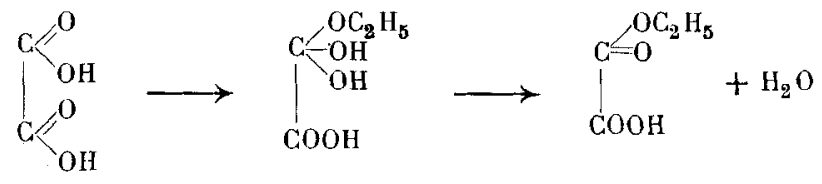

Im allgemeinen ist aber bei den organischen Säuren wenig Neigung vorhanden, die CO-Doppelbindung zu lösen. Eine An-

1) Böttinger, B. 14, 316 (1881).

2) D. R. P. 239 312, Fr. P. 456 156, D. P. Anm. G. 32612 , Kl. 12 o. 
lagerung von Alkohol erfolgt deshalb nur, wenn die Additionsfähigkeit der Carboxyl-CO-Gruppe durch die Neigung der $\alpha$ Wasserstoffatome, sich an Sauerstoff zu fixieren, "zu enolisieren", gesteigert wird. Um das mit Formeln ausdrücken zu können, müsste man etwa schreiben:

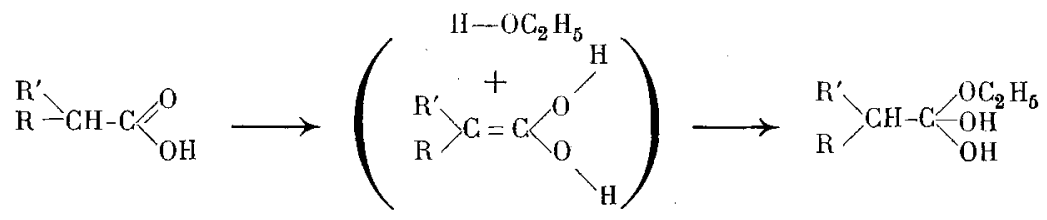

Im Effekt ist das genau dasselbe, wie bei der Oxalsäure. In diesem Falle ist es jedoch das $a$-H-Atom (und nicht die ungesättigte Nachbargruppe), die die CO-Doppelbindung additionsfähig macht, was man gemäss der Formulierung als katalytische Beeinflussung durch das $\alpha$-Wasserstoffatom ansprechen könnte.

Unter dieser Voraussetzung ergibt sich zunächst, dass im allgemeinen eine Säure umso leichter mit einem Alkohol zum Ester zusammentreten wird, je beweglicher ihre Wasserstoffatome in $\alpha$-Stellung zum Carboxyl sind. Das deckt sich vollkommen mit der Erfahrung: Chloressigsäure, Cyanessigsäure, Phenylessigsäure sind wesentlich leichter bezw. rascher esterifizierbar als die Essigsäure selbst. Säuren ohne $\alpha$-ständige Wasserstoffatome sind andererseits extrem leicht in Ester überzuführen, oder gar nicht. Falle der letzteren Art sind in der Fettreihe relativ selten. Dass das Gesagte für die Diäthylmalonsäure zutrifft, ist aus dem experimentellen Teil zu ersehen. Ferner hat Butlerow ${ }^{1}$ ) die Ester der Trimethylessigsäure dargestellt und zwar aus dem Blei- oder Silbersalz mit Halogenalkyl. Leider gibt er nicht an, was ihn dazu veranlasste, diesen umständlicheren Weg zur Darstellung der Ester zu wählen. Ohne das erforderliche Experiment, das mir leider nicht möglich ist, lässt sich also nur vermuten, dass die Trimethylessigsäure genau so wie die Diäthylmalonsäure mit Alkohol keinen Ester liefert.

Als weiteres Beispiel diene noch die von Rupe und Kloppenburg $\left.^{2}\right)$ in jüngster Zeit bestätigte Beobachtung Kachler's, wonach Campholsäure
1) A. $173,371-372(1874)$.
2) Helv. 2, 365-366 (1919). 


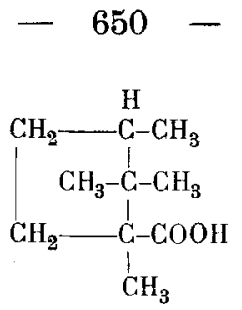

sich mit Alkohol und Salzsäure nicht in den Ester überführen lässt.

Die rein aromatischen Säuren, d. h. die Benzoësäure und ihre Kernsubstitutionsprodukte besitzen gemäss ihrer üblichen Schreibweise<smiles>O=C(O)C1CCCCC1</smiles>

auch kein Wasserstoffatom in $\alpha$-Stellung zum Carboxyl und sind doch im allgemeinen direkt esterifizierbar. Es ist jedoch durchaus denkbar, dass in der Benzoësäure tatsächlich Wasserstoff in Alpha zum Carboxyl steht, wenn auch nur in der halben Zeiteinheit. Es liegt mir ferne, in die Kontroversen über die Konstitution des Benzols einzugreifen. Ich stelle deshalb einfach die allgemein anerkannte Tatsache fest, dass es bei gleichbleibenden Substituenten nur ein einziges o-Disubstitutionsprodukt des Benzols gibt, sodass die allenfalls vorhandenen Doppelbindungen nicht an derselben Stelle stabil verharren können, d. h. die Wasserstoffatome des Benzolkernes sind nicht stabilisiert. Das ergibt die erforderliche Voraussetzung zur Erklärung der Esterifizierbarkeit der Benzoësäure und ihrer Kernsubstitutionsprodukte. Substituenten, die die Beweglichkeit der Wasserstoffatome im Kern erhöhen, werden die Esterifizierbarkeit begünstigen ( $p$-Nitrobenzoësäure ist leichter esterifizierbar als Benzoësäure). Steht ein Substituent in 0-Stellung zum Carboxyl, so wird die Esterbildung erschwert. (Anthranilsäure ist aussergewöhnlich schwer esterifizierbar.) Sind beide ortho-Stellen zum Carboxyl besetzt, so wird kein Wasserstoffatom mehr in $\alpha$-Stellung zum Carboxyl gelangen können; die Esterifizierbarkeit hört ganz auf. 
Diese Tatsache ist bekanntlich von Victor Meyer ${ }^{1}$ ) gefunden und von ihm als sterische Hinderung gedeutet worden. Es sei aber hier noch auf ein weniger bekanntes Detail aus dieser bedeutenden Arbeit hingewiesen. In einer folgenden Abhandlung ${ }^{2}$ ) ist nachgewiesen, dass die o-Substituenten $\mathrm{OH}, \mathrm{CH}_{3}$ oder $\mathrm{NH}_{2}$ noch geringe Veresterung ermöglichen. Victor Meyer nimmt deshalb an, dass die kleineren Substituenten mit geringerem Molekulargewicht sterisch weniger stark hindernd wirken. Im Sinne der hier entwickelten Auffassung wäre aber diese Tatsache so zu verstehen, dass die erwähnten wasserstoff haltigen Gruppen in sehr geringem Masse chinoïde Bindung eingehen können, d. h. es kann trotz eines der erwähnten o-Substituenten ein Wasserstoffatom vorübergehend in $\alpha$-Stellung zum Carboxyl gelangen, gemäss der Formulierung:<smiles>[R]C1CCCC(=O)C1C(=O)O</smiles>

Die Tatsache, dass die Silbersalze der nicht direkt esterifizierbaren aromatischen Säuren mit Halogenalkyl glatt Ester liefern, ist schon Victor Meyer bekannt ${ }^{3}$ ). Mir scheint das ein Beweis gegen seine Anschauung und es ist interessant, den geschraubten Erklärungsversuch an genannter Stelle nachzulesen, auf den hier nur hingewiesen sei.

Mit vorliegenden Ausführungen ist ein erster Versuch zur einheitlichen Erklärung aller bekannten Veresterungs-Anomalien gemacht. Es sei mir noch gestattet, auf eine weitere, aus dieser Anschauung sich ergebende Konsequenz aufmerksam zu machen. Die katalytische Beschleunigung des Esterifizierungsprozesses durch Mineralsäure fasse ich so auf, dass die in alkoholischer Lösung schon an und für sich geringe Ionisation der organischen Säure durch den Mineralsäurezusatz auf ein Minimum heruntergedruckt wird, wodurch die "Enolisierung" begünstigt ist. Die geringste Dissoziation liegt aber zweifellos dann vor, wenn das typische Säurewasserstoffatom durch Alkyl substituiert wird. Ein Ester muss demnach eine labilere CO-Doppelbindung enthalten als die zugehörige freie Säure. Dafür spricht z. B. die leichtere
1) B. 27, $1580(1894)$.
2) B. 28, 1254 (1895).
3) B. 27, 1586 (1894). 
Reduzierbarkeit der Ester gegenüber den freien Säuren. An die durch das $\alpha$-Wasserstoffatom katalytisch beeinflusste CO-Doppelbindung vermag sich aber auch z. B. Ammoniak zu addieren. Unter sekundärer Alkoholabspaltung entsteht aus dem zugehörigen Ester das Säureamid. Die Ursache zur Fähigkeit der Amidbildung aus dem Ester ist also dieselbe wie diejenige der Esterbildung aus Säure und Alkohol. Solche Ester werden also leicht in Amide übergehen, wenn die ihnen zugrundeliegenden Säuren leicht in Ester übergeführt werden können. Das ist, soweit meine Erfahrungen reichen, allgemein richtig. Verblüffend wird die Sache sofort durch die von Emil Fischer ${ }^{1}$ ) festgestellte Tatsache, dass Diäthylmalonester mit Ammoniak kein Amid liefert. Genau so verhält sich der Trimethylessigester ${ }^{2}$. Leider liegt eine analoge Untersuchung der o,o-disubstituierten Benzoësäureester meines Wissens nicht vor.

Experimentelles.

Das Verfahren, das Victor Meyer benützte, um einwandfrei festzustellen, dass o,o-disubstituierte Benzoësäuren mit Salzsäure und Methylalkohol keine Ester liefern, lässt sich im Falle der Diäthylmalonsäure nicht genau analog anwenden. Dieser Autor wies nämlich nach, dass derartige Säuren, die vollkommen rein zu sein schienen, zunächst einige Prozente Ester liefern und erst die regenerierte Säure gar nicht mehr zu verestern ist. Bei der Diäthylmalonsäure tritt aber im Esterifizierungsprozess leicht die Abspaltung einer Carboxylgruppe ein und andererseits kommt das Regenerieren der nicht veresterten Säure als Reinigungsmethode nicht in Betracht, da auch hierbei wieder Kohlendioxydabspaltung eintreten kann. Ich habe mich deshalb darauf beschränkt, eine durch Umkrystallisieren aus Wasser möglichst gereinigte Diäthylmalonsäure zu verwenden. Die erhaltenen Zahlen sprechen aber unter Berücksichtigung dieses erschwerenden Umstandes durchaus dafür, dass eine normale Veresterung bei der Diäthylmalonsäure nicht eintritt, abgesehen davon, dass Diäthylmalonester in zur Identifizierung genügender Menge und Reinheit nicht isoliert werden konnte. Das einzige Umsetzungsprodukt, das bei einem hier nicht anzuführenden Versuch (Veresterung bei $90^{\circ}$ ) identifiziert werden konnte, war Diäthylessigester.
1) B. 35,844 (1902).
3) A. Bannow, B. 35, 856 (1902). 


\section{$-653-$}

\section{Versuch 1}

$100 \mathrm{gr}$ Alkohol $96 \%$, vermischt mit $5 \mathrm{gr}$ Schwefelsäure $92 \%$, und darin bei Zimmertemperatur $80 \mathrm{gr}$ Diäthylmalonsäure durch Umschütteln gelöst. Nach einer halben Stunde ist völlige Lösung eingetreten. Darauf wurde 24 Stunden im Wasserbad von $50-55^{01}$ ) erwärmt und die Reaktionsmasse in einen halben Liter kaltes Wasser eingerührt. Es entstand nur eine geringe milchige Trübung; eine Ölschicht kam nicht zur Abscheidung.

\section{Versuch 2.}

100 gr Alkohol $96 \%$, vermischt mit 5 gr Schwefelsäure $92 \%$, und darin bei Zimmertemperatur $52 \mathrm{gr}$ Malonsäure durch Umschütteln gelöst. Nach einer halben Stunde ist völlige Lösung eingetreten.

Darauf wurde 24 Stunden im Wasserbad von 50-55 erwärmt und die Reaktionsmasse in einem halben Liter kaltes Wasser eingerührt. Das in reichlicher Menge sich unten ansammelnde Öl wurde im Scheidetrichter abgezogen und mit Sodalösung gewaschen, und alsdann im Vakuum destilliert. Ausbeute an Malonester $55 \mathrm{gr}=70 \%$ der Theorie.

\section{Versuch 3.}

In $12,5 \mathrm{~cm}^{3}$ einer 14 Tage lang in geschlossener Flasche gestandenen alkoholischen Schwefelsäure (300 gr Alkohol $96 \%+$ $15 \mathrm{gr} \mathrm{H}_{2} \mathrm{SO}_{4} 92 \%$ ) wurden $10 \mathrm{gr}$ Diäthylmalonsäure bei gewöhnlicher Temperatur durch Umschütteln gelöst.

$5 \mathrm{~cm}^{3}$ dieser Lösung (ca. 21,0 $\mathrm{cm}^{3}$ Gesamtmenge) sofort nach erfolgter Lösung mit Wasser verdünnt, verbrauchten zur Neutralisation (Phenolphtaleïn als Indikator) $28,05 \mathrm{~cm}^{3}$ n.KOH und nach 24-stündigem Erwärmen im Wasserbad von $50^{\circ} 27,30 \mathrm{~cm}^{3}$ n.KOH. Die Differenz beträgt also $0,75 \mathrm{~cm}^{3} \mathrm{n} . \mathrm{KOH}=2,5 \%$ berechnet auf Diäthylmalonsäure.

\section{Versuch 4.}

In $12,5 \mathrm{~cm}^{3}$ ca. normaler alkoholischer Salzsäure (Alkohol $96 \%$ ) wurden 10 gr Diäthylmalonsäure bei gewöhnlicher Temperatur durch Umschütteln gelöst.

1) Bei wesentlich höherer Temperatur wird Kohlendioxyd abgespalten. 
$5 \mathrm{~cm}^{3}$ dieser Lösung (ca. 21,0 $\mathrm{cm}^{3}$ Gesamtmenge) sofort nach erfolgter Lösung mit Wasser verdünnt, verbrauchten zur Neutralisation (Phenolphtaleïn als Indikator) $30,25 \mathrm{~cm}^{3} \mathrm{n} . \mathrm{KOH}$ und nach 24-stündigem Erwärmen im Wasserbad von $50^{\circ} 27,90 \mathrm{~cm}^{3}$ n.KOH. Die Differenz beträgt also $2,35 \mathrm{~cm}^{3} \mathrm{n} . \mathrm{KOH}=7,7 \%$, berechnet auf Diäthylmalonsäure.

\section{Versuch 5.}

In $21 \mathrm{gr}$ alkoholischer Schwefelsäure (300 gr Alkohol + $15 \mathrm{gr}$ $\mathrm{H}_{2} \mathrm{SO}_{4}$ ) wurden bei gewöhnlicher Temperatur 10,4 gr Malonsäure unter Umschütteln gelöst.

$5 \mathrm{~cm}^{3}$ dieser Lösung (ca. $32,8 \mathrm{~cm}^{3}$ Gesamtmenge) sofort nach erfolgter Lösung mit Wasser verdiunnt, verbrauchten zur Neutralisation (Phenolphtaleïn als Indikator) $28,68 \mathrm{~cm}^{3}$ n.KOH und nach 24-stündigem Erwärmen im Wasserbad von $50^{\circ} 8,38 \mathrm{~cm}^{3}$ n.KOH. Die Differenz beträgt also $20,3 \mathrm{~cm}^{3} \mathrm{n} . \mathrm{KOH}=66,7 \%$, berechnet auf Malonsäure.

Dass diese Zahl niedriger ist als die in Versuch 2 effektiv erhaltene Ausbeute an Malonester rührt wohl davon her, dass schon während des Lösens Malonsäure der Veresterung anheimfällt. Da aber die Esterifizierbarkeit der Malonsäure zur Genüge bekannt ist, wurde von einer näheren Festlegung der Umsetzungsgeschwindigkeit abgesehen.

Basel, Wissenschaftliches Laboratorium der Firma

F. Hoffmann-La Roche \& Co. A.G.

September 1919. 